\title{
High Resolution Electron Microscopy - Principles and Practice
}

\author{
David J. Smith*
}

* Center for Solid State Science and Department of Physics and Astronomy, Arizona State University, Tempe, AZ 85287

Most transmission electron microscopes sold commercially nowadays are capable of resolving atomic-level detail in suitably thinned samples but there are many potential pitfalls along the way for the budding high-resolution electron microscopist. Some basic knowledge of beam-specimen interactions and some understanding of contrast transfer theory are essential to ensure correct microscope operation. This tutorial will provide an overview of high-resolution electron microscopy, beginning with basic theory and instrumentation requirements, and then proceeding to details of imaging and image interpretation. Possible sources of image artefacts and requirements for sample preparation are briefly discussed.

Because it is specimen-independent, the contrast transfer function (CTF) provides a convenient mechanism for understanding the actual imaging process inside any objective lens, and the use of (dimensionless) universal curves allows for easy interpolations and comparisons for different microscopes, i.e. effect of $\mathrm{Cs}_{\mathrm{s}} \mathrm{kV}$, etc. For coherent, axial illumination, the CTF for weak phase object imaging can be represented by the expression: $\sin \left\{\pi \mathrm{k}^{2}\left(\mathrm{k}^{2} / 2-\mathrm{D}\right)\right\}$, where $\mathrm{k}$ and $\mathrm{D}$ are, respectively, the spatial frequency and the objective lens defocus in generalized coordinates [1]. In the "real" world, the CTF is likely to be affected by beam divergence (spatial coherence) and focal spread (temporal coherence), which will dampen out higher spatial frequencies (higher-resolution detail). It is therefore important to limit the beam divergence with a suitable condenser aperture (typical values are 0.3 to $0.6 \mathrm{mrad}$ ), and to restrict the beam energy spread by using low emission currents (no more than $\sim 2-3 \mu \mathrm{A}$ at $200-400 \mathrm{keV}$ ). Use of a high coherence, field emission gun (FEG) electron source will be less limiting in these aspects but the extra CTF oscillations (which can be avoided with a suitable objective aperture) will conversely complicate the image interpretability.

Instrumental parameters important for HREM operation include: the beam energy spread, the illumination divergence, the objective lens defocus and spherical aberration coefficient. The latter are strongly dependent on the objective lens current. Thus, it is good practice to determine their values relative to a particular OL current and thereafter keep this current fixed and then adjust the specimen height ("Z"-lift) to bring the image close to focus. Of course, the objective lens astigmatism is important but since it should ideally be adjusted to zero then it is not normally considered necessary to determine its absolute value. Information about measurement of these various key parameters can be found elsewhere [1]. Extraneous factors such as mechanical vibrations, acoustic noise and stray $\mathrm{AC}$ fields can normally be avoided by careful choice of the microscope location BUT regular checks with standard samples (such as small metal particles on a thin amorphous film) is nevertheless recommended as good operating practice.

The basic configuration and adjustment of the TEM for high-resolution phase contrast imaging is identical to that used for diffraction contrast. The gun tilt should be adjusted for optimum emission, and the gun/condenser lens system should be aligned optimally. The desired condenser aperture(s) should be clean and centered, and the objective aperture(s) should also be kept clean to avoid image 
astigmatism. The most important adjustments relate to the objective lens. Accurate defocus is required, which can be best obtained by locating the minimum contrast focus and then offsetting by the pre-determined (calibrated?) focal steps. The objective lens astigmatism should be adjusted by reference to a nearby region of amorphous material (use the on-line diffractogram capability if available!). After checking the beam-tilt purity adjustments, the incident beam direction should be adjusted to minimize off-axis coma. (Note that small amounts of beam tilt will cause images and diffractograms that resemble astigmatism.) The best method of beam alignment for the unaided operator is by reference to the appearance of amorphous films in successive tilted-beam images using a beam-tilt "wobble" [2]. Specimen tilts for crystalline samples need to be carefully adjusted so that the incident beam is aligned along the desired zone axis - with this adjustment becoming more critical at higher resolution and for thicker specimen regions. The exact choice of image magnification is not really critical. There is always a temptation to use excessive magnification in order to see finer image details BUT the undesirable consequences are likely to be increased beam divergence (resolution limiting?) and greater likelihood of beam damage. Conversely, lower magnification operation makes focusing difficult and will degrade signal statistics. Operation in the range of $400,000 x$ to 750,000 is usually adequate for recording atomic-resolution images using photographic film or CCD cameras.

High-resolution operation and image interpretation are complicated by the rapid and often complex variations in image appearance with changes in defocus and thickness, as well as the dependence on $\mathrm{Cs}_{\text {s }}$ and $\mathrm{kV}$. For extracting quantitative high-resolution information, image simulation is highly desirable, particularly for interpreting detail close to the structural resolution limit. Simulations are absolutely essential for detail beyond this limit. The inexperienced operator needs to be aware of other possible imaging artefacts. Fourier or "self" images of crystalline materials recur with defocus, with a period that depends on unit-cell size and shape, as well as electron wavelength. A nearby crystal edge or amorphous region should normally be utilized to locate the "in-focus" minimum contrast position for reference purposes. "Atomic-pair" or "dumbell" images can be obtained from small-unit-cell semiconductors, at least in very thin regions, under very stringent operating conditions when the microscope resolution closely approaches $1 \AA$. However, these commonly observed features are oftentimes complex interference effects originating from thicker regions which reflect the crystal symmetry and the instrumental stability and they cannot usually be directly related to specific atomic positions.

Sample preparation becomes more and more demanding as resolution limits improve. Conventional ion-beam milling for cross-sectional samples will likely contribute amorphous overlayers unless the final milling is done at low ion-beam energy $(1-2 \mathrm{keV})$ and incident angle (4-6 ${ }^{\circ}$. Focused-ion-beam milling is becoming commonplace for complicated specimen geometries but this approach to sample thinning is actually far worse in terms of contributing amorphous overlayers that will obscure the finest specimen detail that is of most interest. Wedge polishing and very low energy $(<1 \mathrm{keV})$ ionmilling are thus becoming more prevalent in laboratories seeking the highest possible specimen resolution.

[1] D.J. Smith, Adv. Opt. El. Micr. 11 (1989) 1

[2] D.J. Smith, et al., Ultramicroscopy 11 (1983) 263. 